\section{A computer program for automated scoring of exteroceptive suppression periods}

\author{
DOUGLAS J. FRENCH \\ Université de Moncton, Moncton, \\ New Brunswick, Canada \\ and
}

CHRISTOPHER R. FRANCE, KENNETH A. HOLROYD, and ANUJ G. MEHTA

Ohio University, Athens, Ohio

Exteroceptive suppression (ES) of temporalis muscle activity has received recent attention as a method of investigating central mechanisms in the pathogenesis of chronic headache disorders. Unfortunately, the resolution of conflicting findings has been hampered by the use of different assessment and scoring methodologies across laboratories, which has limited the direct comparison of findings across studies. A computer program was developed with the goal of providing an automated and reliable method of scoring ES responses. Processing an ES response with this software provides both visual and quantitative information relating to various response parameters. Multiple levels of smoothing the raw waveform data are available, and the software offers scoring on the basis of multiple depth-of-suppression criteria. The program also generates values for a variety of ES parameters: latency, duration, area, maximum level of suppression with its corresponding time ( $\mathrm{msec}$ ), and baseline level of muscle activity. It is likely that a reliable and standardized scoring protocol would enhance comparability of ES data generated across different settings.

Exteroceptive suppression (ES) of temporalis and masseter muscle activity has received increasing attention as a method of investigating central mechanisms in the pathogenesis of chronic headache disorders. Normally, perioral electrical stimulation during voluntary jaw occlusion elicits two successive suppression periods (ES1 \& ES2). Although a series of studies have confirmed an attenuated or absent ES2 in chronic tension headache subjects relative to headache-free controls (Nakashima \& Takahashi, 1991; Paulus, Raubüchl, Straube, \& Schoenen, 1991; Schoenen, Jamart, Gerard, Lenarduzzi, \& Delwaide, 1987), there have also been negative findings (Bendtsen, Jensen, Brennum, Arendt-Nielsen, \& Olesen, 1996; Lipchik et al., 1996; Lipchik, Holroyd, Talbot, \& Greer, 1997; Zwart \&

Support for this research was provided by an Academic Challenge Award from the State of Ohio. Please note that a new stand-alone version of the program has been recently developed using visual basic. This new version employs the same ES scoring algorithms and offers users similar features as those of the program described in the present manuscript yet does not require Excel. Requests for reprints or copies of the program should be sent to D. J. French, Département de Psychologie, Université de Moncton, Moncton, NB, ElA 3E9 Canada (e-mail: frenchdo@umoncton.ca).
Sand, 1995). Because stimulation parameters used to elicit the ES response (e.g., frequency, intensity, and duration of stimulation) and the procedures for quantifying the resultant suppression periods have varied widely across studies from different laboratories (Bendtsen, Jensen, Brennum, Arendt-Nielson, \& Olesen, 1993, 1996; Lipchik et al., 1996; Lipchik et al., 1997; Nakashima \& Takahashi, 1991; Paulus et al., 1991; Schoenen et al., 1987; Wallasch, Niemann, Kropp, \& Weinshütz, 1993; Zwart \& Sand, 1995), findings often cannot be directly compared across studies.

In an attempt to standardize methodologies, a series of papers (Göbel, Dworschak, \& Wallasch, 1993; Schoenen, 1993; Wallasch \& Göbel, 1993) examined previous ES findings and yielded a number of methodological recommendations to guide future investigations of this neurophysiological response (Göbel \& Schoenen, 1993). Although these recommendations appear to have led to increased standardization in the methods used to elicit ES in subsequent studies, no clear consensus has emerged regarding a uniform method for quantifying ES responses once they are recorded.

Nakashima and Takahashi (1991) calculated latency and duration of suppression at the point where the electromyographic (EMG) tracing crossed the mean prestimulation baseline - in other words, a $0 \%$ suppression criteria. Göbel and Schoenen (1993) recommended that an $80 \%$ reduction in EMG activity, relative to activity recorded $20 \mathrm{msec}$ before stimulation, be adopted as the criterion for identifying suppression periods. Although some have adopted an $80 \%$ reduction in EMG activity criterion for measuring suppression, baseline level of EMG activity has been calculated using both prestimulation (Wang, De Pasqua, Gerard, \& Schoenen, 1995) and poststimulation muscle activity (Göbel et al., 1994). Finally, Bendtsen et al. (1993), in an investigation of ES variability, found greater inter- and intrasubject ES variability among headache-free subjects when they defined exteroceptive suppression as a reduction below $80 \%$ baseline EMG activity, as compared with a $50 \%$ suppression criterion. These authors also reported a large number of absent ES2 responses in their "healthy" sample, suggesting that an $80 \%$ suppression criterion may be too stringent for identifying abnormal responses. If ES latencies and durations continue to vary across settings because of differences in methodology, comparisons across studies will continue to be difficult and unreliable.

The present manuscript describes a computer program that was written using Microsoft Excel macro language in the hopes of providing researchers a simple, objective, and reliable method of scoring digitized EMG data obtained in the assessment of ES responses. Attempts to calculate ES parameters, using automated and semi-automated scoring procedures, have been reported previously (Bendtsen et al., 1993, 1996; van der Glas \& van Steenberghe, 1981).

An ambitious ES scoring program was developed by van der Glas and van Steenberghe (1981) that would auto- 
matically generate a large number of ES parameters from digitally recorded EMG activity. Unfortunately, many of the ES parameters generated by the program, such as the latency of the highest post-ES2 voltage, are currently not considered important in the understanding or the analysis of the ES response. Similarly, the ES assessment methods on which the van der Glas and van Steenberghe program is based are no longer in use. For example, recording of EMG responses was triggered mechanically by a swinging pendulum used to stimulate or "tap" subjects incisor tooth pulp - a method that has since been abandoned in favor of more reliable electrical stimulation. This methodology also precluded recording pre- and poststimulation EMG activity on the same waveform, as they were necessarily assessed separately. Furthermore, levels of suppression were not automatically expressed in percent change from prestimulus EMG levels, which has since become the recommended methodology (Göbel \& Schoenen, 1993) to facilitate comparisons across research sites.

Bendtsen et al. (1993, 1996) describe a semi-automated scoring program that generates an ES waveform from raw EMG data and superimposes horizontal suppression threshold lines representing $50 \%$ and $80 \%$ of mean prestimulation EMG activity. In order to calculate onset and duration of ES periods using this method, the experimenter must manually place vertical cursors at points on the tracing where the waveform crosses the threshold lines. The program generates the EMG waveform and relative suppression thresholds once the cursors have been adjusted. However, the determination of ES onset and latency are ultimately determined by the experimenter's subjective visual inspection of the waveform and is, therefore, vulnerable to experimenter bias.

The present scoring improves upon existing systems by adhering to standardized assessment methodology, by eliminating the potential for experimenter bias by fully automating the calculation of all ES parameters, and, finally, by flexibly offering options to manually generate some or all suppression values, if desired.

\section{DATA ENTRY}

The current version of the software will read input data saved in standard ASCII format. The only restrictions are that data are collected with the use of established procedures (e.g., $250 \mathrm{msec}$ of ASCII data sampled at $2 \mathrm{KHz}$, with stimulation occurring at $75 \mathrm{msec}$ ) and that EMG voltage values and associated recording times are stored in matched columns.

The software may be configured to process data from a variety of data collection systems. User-defined values are entered to (1) identify the location of voltage and time data in the ASCII file, (2) convert EMG voltage values into microvolts $(\mu \mathrm{V})$ for signal calibration, and (3) define the temporal search interval for identifying the location of ES responses. Default temporal search intervals are from 5.5 to $35.5 \mathrm{msec}$ poststimulus for locating the ES1 maximum suppression point and from 36 to $106 \mathrm{msec}$ for locating the ES2 maximum suppression point. Configura- tion of the software need only be performed once, unless modifications of data collection procedures necessitate updating the existing parameters.

\section{DATA PROCESSING}

When a new data file is opened from within the computerized scoring program using the default settings, a graphical display of the ES profile is automatically generated in the following manner: (1) An average prestimulation baseline EMG value is calculated from the 100 EMG values $(\mu \mathrm{V})$ that comprise the 50-msec interval immediately preceding electrical stimulation; (2) all EMG values collected during the entire $250 \mathrm{msec}$ of recording ( $75 \mathrm{msec}$ prestimulation, $175 \mathrm{msec}$ poststimulation) are then converted into a percentage of the prestimulation baseline EMG calculated in Step 1; and (3) these percentage values are then graphed across time $(250 \mathrm{msec})$. Figure 1 provides an example of an ES waveform generated using raw rectified EMG and the corresponding waveform generated by the scoring program, expressed as percent change from baseline. As is shown in Figure 1, converting the raw EMG values to a percent change from baseline value does not alter the shape of the waveform but does facilitate visual inspection by allowing the addition of threshold lines indicating $0 \%, 50 \%$, and $80 \%$ suppression levels.

On the basis of a procedure reported by Göbel and colleagues (1994), the scoring program offers an option that calculates the baseline EMG value (and subsequent percent change values), using poststimulus rather than prestimulus EMG activity. With this method, the highest poststimulation EMG value is first identified by the program. Next, all poststimulation EMG values falling within a defined percentage of the maximum value are averaged (Göbel and colleagues chose $30 \%$ ). This average EMG value is then used as the baseline for determining suppression thresholds. When this option is selected, the user can define the percentage value used for calculating the poststimulation baseline. For example, the user may input any percentage value from 1 to 100 . The program then averages all EMG values that fall within the specified percentage range and calculates the baseline on the basis of the resulting poststimulation EMG average.

The present software also has an option that will allow the user to specify any recorded EMG value as the baseline. Once a file is opened, the user can scroll along the waveform, using a movable cursor, stop at any point, and designate the corresponding EMG value as the baseline. This value would then be entered manually, and the ES parameters recalculated on the basis of the new baseline. Separate baselines can be generated for ES1 and ES2, which may be useful in the event of a stimulus artifact that may have affected only ES1 because of its temporal proximity to the electrical stimulation.

The user has the option of using either smoothed or unsmoothed EMG values in the calculation of ES parameters. The user may select unsmoothed data (i.e., ES parameters based on the raw rectified EMG values) or one of 

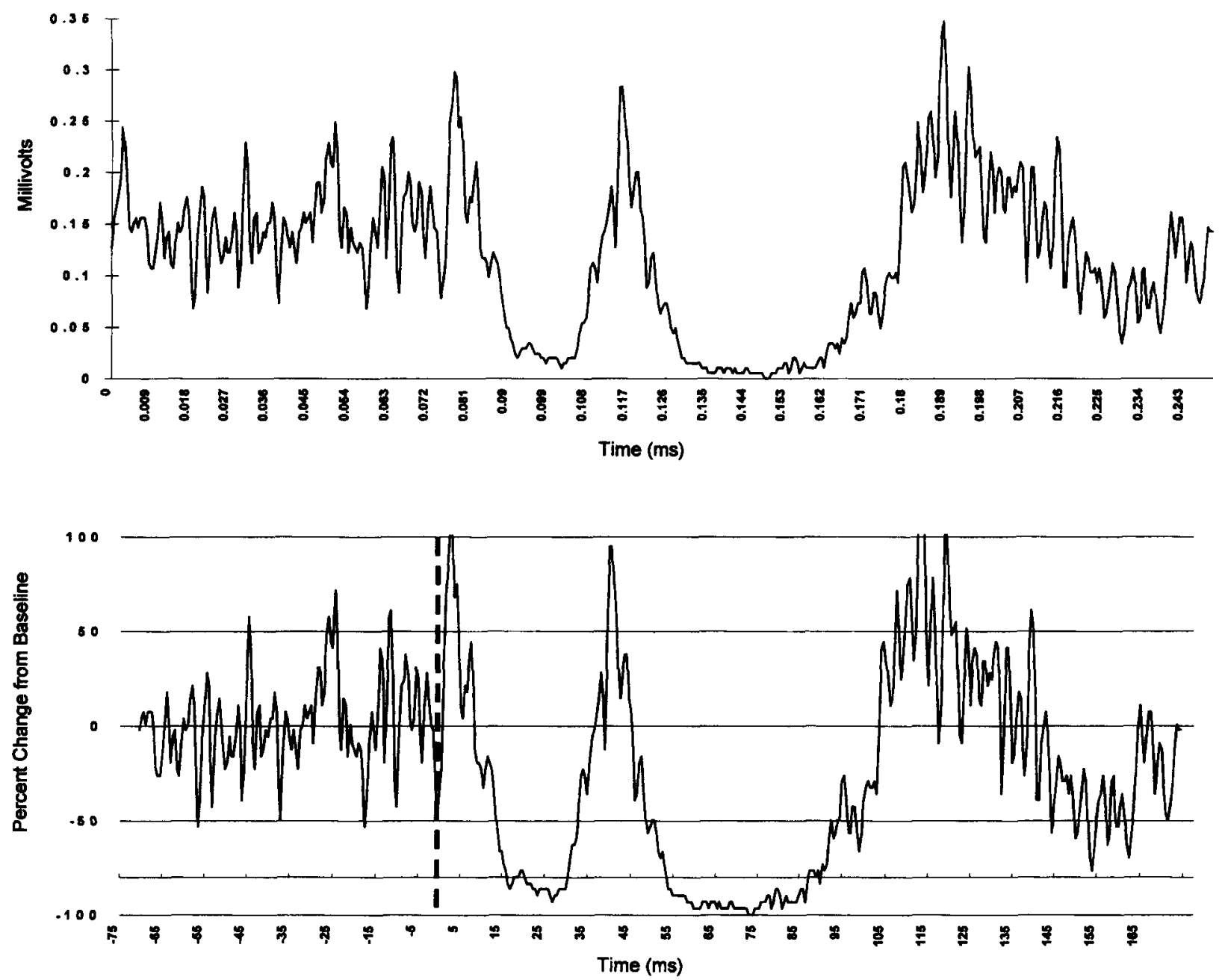

Figure 1. Waveform of averaged, rectified EMG (expressed in millivolts) and percent change from prestimulus baseline.

two levels of smoothing (5- or 10-point smoothing-a running average with each EMG value expressed as a average of the two or five values before and after, respectively). Figure 2 shows the 10-point smoothed ES response presented in Figure 1. Smoothing the waveform facilitates accurate judgments regarding onset and offset of suppression periods by removing transient crossings of the criterion suppression threshold.

\section{Automated Scoring}

The primary user interface screen is presented in Figure 3. Virtual "buttons" are used to select level of smoothing and to perform operations, such as opening a file, viewing a graph, initiating automated and/or manual scoring, and copying ES parameters to a table that may be viewed, saved to disk, or printed. As is shown in Figure 3, all ES parameters are clearly labeled at each level of suppression for both automated and manual scoring procedures. The user also may select the method for calculating baseline EMG and the scoring method from the primary interface screen.
The software automatically identifies ES1 and ES2 suppression periods and generates values for ES parameters. Onset (latency), offset (end point), duration, and area are calculated, using each of the three depth of suppression criteria $(0 \%, 50 \%, 80 \%)$, with the following routines: (1) The program identifies the lowest EMG value within each ES1 and ES2 temporal search interval; (2) simultaneously scrolling backward and forward along the waveform, the program identifies the time at which the waveform intersects each of the three suppression threshold lines $(0 \%, 50 \%$, and $80 \%)$. For example, using a $50 \%$ depth of suppression criterion, latency and end point represent the times at which the waveform values descend and ascend, respectively, $50 \%$ of the prestimulus baseline (represented by a line on the graph; see Figure 2). Duration at $50 \%$ depth of suppression criterion would be the amount of time the suppression period remained below the 50\% line. Area represents the sum of all values below the threshold level at each level of suppression. Finally, maximum level of suppression, with its corresponding time (msec), and baseline level of muscle activity $(\mu \mathrm{V})$ are also provided. 


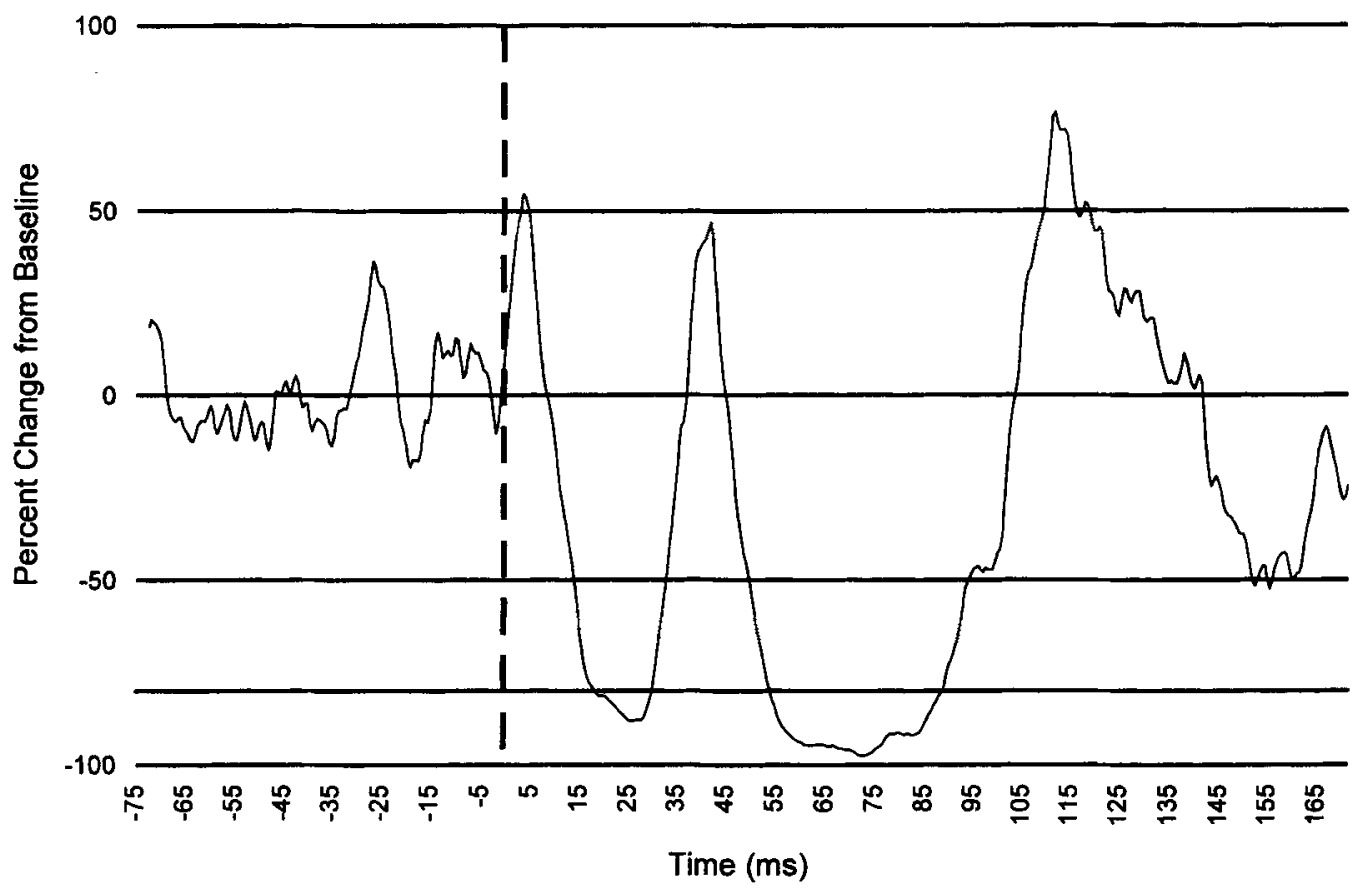

Figure 2. Ten-point smoothed waveform of averaged rectified EMG.

In the relatively rare case of a profile with a "merged" ES1 and ES2 (i.e., EMG activity fails to recover above the suppression threshold between ES1 and ES2), EMG activity suppressions are automatically identified and scored appropriately. The program does this by using the following procedure: EMG values are examined between the lowest value identified in each of the ES1 and ES2 search intervals. If there is no value greater than a given threshold level, the response is flagged as merged at that level of suppression. In these cases, the highest point between the minimum values identified in both the ES1 and the ES2 search intervals is chosen as the endpoint of ES1 and the onset of ES2. Using these merged values, ES parameters are automatically generated by the algorithm used by the program. This procedure automates an algorithm reported by van der Glas and van Steenberghe (1981).

\section{Manual Scoring}

The program can also facilitate semi-automated "manual" scoring. This involves the entry of user-defined latency and end point values for ES1 and ES2 responses that are derived by scrolling along the waveform, using a moving onscreen cursor. The user then determines the times associated with the selected points on the waveforms which are subsequently entered into the primary user interface. The program calculates the remaining parameters, using the entered values. Manual scoring may be required for poorly recorded or otherwise unusual recordings. This procedure essentially duplicates the program described by Bendtsen et al. (1993).

\section{Output}

The data generated by both automatic and manual scoring methods may be copied onto a summary table for sub- sequent storage and printing. Results may be saved and printed in graphic, tabular, and ASCII formats. Data saved in ASCII format may be directly imported into other programs for subsequent statistical analysis.

\section{CONCLUSIONS}

The present software allows reliable, standardized, and precise quantification of ES periods and thus has the potential to increase the comparability of findings obtained by different investigators. An automated scoring system has the advantages of eliminating any influence of experimenter bias on manual scoring and of allowing the rapid generation of characteristics (e.g., area) of ES periods that may be difficult or impossible to accurately calculate manually. An automated scoring system would thus facilitate the identification of the optimum combination of scoring parameters (i.e., suppression threshold, smoothing factor) and the optimum suppression parameter (duration, area) for various purposes. For example, the scoring and suppression parameters that best discriminates between tension-type headache disorders and other headache disorders.

Göbel and Schoenen (1993) have suggested that an $80 \%$ reduction in EMG activity is the preferred criteria for identification of the onset of a suppression period, whereas Bendtsen et al. (1993) have suggested that a 50\% reduction in EMG activity is likely to be a better criterion. To date, however, no effort has been made to empirically compare different suppression criteria or different indices of suppression for their ability to identify diagnostic groups. The availability of an easy-to-use automated software for the identification and scoring of ES responses should facilitate such comparisons. 


\begin{tabular}{|c|c|c|c|c|c|c|}
\hline \multicolumn{4}{|c|}{ Exteroceptive Suppression Scoring } & \multicolumn{2}{|c|}{ FILENAME 1001_t1 } & \multirow{2}{*}{$\begin{array}{l}\text { SMCOTH } \\
\text { ГACTOR }\end{array}$} \\
\hline AUIUMAIEU & & & \multirow{2}{*}{ MANUAL } & & & \\
\hline & ES-1 & ES-2 & & ES-1 & ES-2 & D \\
\hline LATENCY $(0 \%)$ & 8.25 & 45.25 & LATENCY $(0 \%)$ & $<-$ & $<-$ & \\
\hline END POINT (0\%) & 37.25 & 103.75 & END POINT $(0 \%)$ & $<-$ & $<-$ & 5 \\
\hline DURATION $(0 \%)$ & 29 & 58.5 & DURATION $(0 \%)$ & & & \\
\hline $\begin{array}{ll}\text { AREA } & (0 \%) \\
\end{array}$ & -3433.71 & -8681.89 & $\begin{array}{ll}\text { AREA } & (0 \%) \\
\end{array}$ & & & 10 \\
\hline & & & & CLEAR & CLEAR & \multirow{3}{*}{$\begin{array}{l}\text { OPEN } \\
\text { FILE }\end{array}$} \\
\hline \multirow[t]{2}{*}{ MAX } & -88.26 & -97.53 & \multirow[t]{2}{*}{ MAX } & & & \\
\hline & & & & CLEAR & CLEAR & \\
\hline LATCNCY $(50 \%)$ & 13.75 & 49.75 & LATENCY (50\%) & $<-$ & $\leqslant-$ & \\
\hline END POINT $(50 \%)$ & 32.75 & 94.25 & END POINT (50\%) & $<-$ & $<-$ & $\begin{array}{l}\text { GRAPH } \\
\text { VIEW }\end{array}$ \\
\hline DURATION $(50 \%)$ & 19 & 44.5 & DURATION $(50 \%)$ & & & \multirow{3}{*}{$\begin{array}{l}\text { AUTO } \\
\text { CALC. }\end{array}$} \\
\hline \multirow[t]{2}{*}{ ARtA $\quad$ (b0\% } & $-10 / 6.39$ & -3256.63 & AREA $\quad(50 \%)$ & & & \\
\hline & & & & CIFAR & CAFAR & \\
\hline LATENCY (80\%) & 18.25 & 53.75 & UATENCY (80\%) & $<-$ & $<$ & \multirow{2}{*}{$\begin{array}{l}\text { MANUAL } \\
\text { CALC. }\end{array}$} \\
\hline END POINT( $80 \%)$ & 29.76 & 88.76 & \multirow{2}{*}{ DURATION (80\%) } & $<-$ & $\Leftrightarrow$ & \\
\hline DURATION ( $30 \%)$ & 11.5 & 35 & & & & \multirow{2}{*}{$\begin{array}{l}\text { COPY TO } \\
\text { SHEET }\end{array}$} \\
\hline \multirow[t]{2}{*}{ AREA $\quad(80 \%)$} & -112.99 & -859.86 & AREA $\quad(80 \%)$ & & & \\
\hline & EY & & & & & \multirow{2}{*}{$\begin{array}{l}\text { VIEW } \\
\text { SHEET }\end{array}$} \\
\hline PRESTIMULUS & 161.83 & & \multirow{3}{*}{$\begin{array}{c}\text { CLEAR } \\
\text { MANUAL }\end{array}$} & \multirow{3}{*}{$\begin{array}{c}\text { USER } \\
\text { DEFINED } \\
\text { BASFI INF }\end{array}$} & \multirow{3}{*}{$\begin{array}{l}\text { REDO } \\
\text { MANUAL } \\
\text { CAI } \mathrm{C}\end{array}$} & \\
\hline $\begin{array}{l}\text { USER DEFINED } \\
\text { ES1 BASELINE }\end{array}$ & & TIMEinse & & & & EXIT \\
\hline FS? RASFIINF & & & & & & \\
\hline
\end{tabular}

Figure 3. Primary user interface showing computer-generated suppression parameters.

\section{REFERENCES}

Bendtsen, L., Jensen, R., Brennum, J., ARendt-Nielsen, L., \& OleSEN, J. (1993). Exteroceptive suppression periods in jaw-closing muscles: Variability and relation to experimental pain and sustained muscle contraction. Cephalalgia, 13, 184-191.

Bendtsen, L., Jensen, R., Brennum, J., Arendt-Nielsen, L., \& OleSEN, J. (1996). Exteroceptive suppression of temporal muscle activity is normal in chronic tension-type headache and not related to actual headache state. Cephalalgia, 16, 251-256.

Göbel, H., Dworschak, M., \& Wallasch, T.-M. (1993). Exteroceptive suppression of temporalis muscle activity: Perspectives in headache pain and research. Cephalalgia, 13, 15-19.

Göbel, H., Krapat, S., Dworschak, M., Heuss, D., Ensink, F. B. M., \& SOYKA, D. (1994). Exteroceptive suppression of temporalis muscle activity during migraine attack and migraine interval before and after treatment with sumatriptan. Cephalalgia, 14, 143-148.

Göbel, H., \& SChOENEN, J. (1993). Exteroceptive suppression in headache research: Discussion summary. Cephalalgia, 13, 20.

Lipchik, G. L., Holroyd, K. A., France, C. R., Kvaal, S. A., Segal, D., Cordingley, G., Rokicki, L. A., \& McCool, H. R. (1996). Central and peripheral mechanisms in chronic tension-type headache. Pain, 64, 467-475.

Lipchik, G. L., Holroyd, K. A., TAlbot, F., \& Greer, M. (1997). Pericranial muscle tenderness and exteroceptive suppression of temporalis muscle activity: A blind study of chronic tension-type headache. Headache, 37, 368-376

NAKASHIMA, K., \& TAKAHASHI, K. (1991). Exteroceptive suppression of the masseter, temporalis and trapezius muscles produced by mental nerve stimulation in patients with chronic headaches. Cephalalgia, 11, 23-28.
Paulus, W., Raubüchl, O., Straube, A., \& Schoenen, J. (1991). Exteroceptive suppression of temporalis muscle activity in various types of headache. Headache, 32, 41-44.

SCHOENEN, J. (1993). Exteroceptive suppression of temporalis muscle activity: Methodological and physiological aspects. Cephalalgia, 13, 3-10.

Schoenen, J., Jamart, B., Gerard, P., Lenarduzzi, P., \& Delwaide, P. J. (1987). Exteroceptive suppression of temporalis muscle activity in chronic headache. Neurology, 37, 1834-1836.

van der Glas, H. W., \& van Steenberghe, D. (1981). Computerbased analysis of electromyographic silent period parameters in jaw muscles during clenching in man. Electromyography \& Clinical Neurophysiology, 21, 627-641.

WALLASCH, T.-M., \& GöBEL, H. (1993). Exteroceptive suppression of temporalis muscle activity: Findings in headache. Cephalalgia, 13, $11-14$.

Wallasch, T.-M., NiemanN, U., Kropp, P., \& Weinshütz, T. (1993). Exteroceptive silent periods of temporalis muscle activity: Correlation with neuropsychological findings. Headache, 33, 121-124.

Wang, W., De Pasqua, V., Gerard, P., \& Schoenen, J. (1995). Specificity and sensitivity of temporalis ES2 measurements in the diagnosis of chronic primary headaches. Headache, 35, 85-88.

ZWART, J.-A., \& SAND, T. (1995). Exteroceptive suppression of temporalis muscle activity: A blind study of tension-type headache, migraine and cervicogenic headache. Headache, 35, 338-343.

(Manuscript received March 14, 1997; revision accepted for publication August 22, 1997.) 